\title{
Cloning and prokaryotic expression of rat homolog of Serpina3n and its expression change during liver regeneration
}

\author{
G.P. Wang ${ }^{1 *}$, X.S. Zhang ${ }^{1 *}$, Y.H. Li ${ }^{2}$, J.L. Zheng ${ }^{1}$, C.Z. Tang ${ }^{1}$ and \\ W.X. Zhang ${ }^{1}$ \\ ${ }^{1}$ College of Life Sciences, Henan Normal University, Xinxiang, \\ Henan Province, China \\ ${ }^{2}$ Department of Life Science and Technology, Xinxiang Medical University, \\ Xinxiang, Henan Province, China \\ *These authors contributed equally to this study. \\ Corresponding author: Y.H. Li \\ E-mail: yonghaili@126.com
}

Genet. Mol. Res. 11 (3): 3175-3185 (2012)

Received February 15, 2012

Accepted August 3, 2012

Published September 3, 2012

DOI http://dx.doi.org/10.4238/2012.September.3.6

\begin{abstract}
A strikingly upregulated expressed sequence tag was screened from regenerating rat liver at $8 \mathrm{~h}$ in a $0-4-8-12 \mathrm{~h}$ short-interval successive partial hepatectomy model from a previous study. In the present study, a full-length open reading frame (ORF) corresponding to this expressed sequence tag was predicted through electronic cloning and was subsequently cloned from an 8-h rat regenerating liver and deposited in GenBank (accession No. HM448398). Sequence analysis of HM448398 and the predicted ORF revealed that the two ORFs may be different transcripts of a gene. The sequence of HM448398 was highly homologous to that of rat Serpina3n, suggesting that it may be a homolog of Serpina3n. The pGEX-2TK prokaryotic expression vector for this ORF was constructed, and the result of sodium dodecyl sulfate polyacrylamide gel electrophoresis manifested that the recombinant expression vector could express the glutathione-S-transferase-fused rat homolog of Serpina3n in an insoluble form in BL21. The target fusion
\end{abstract}


protein was purified with affinity chromatography and was used as antigen to immunize rabbits for the production of polyclonal antibodies. Immunohistochemistry and real-time reverse transcription polymerase chain reaction analysis revealed that the gene was highly expressed in the priming and termination phases of liver regeneration. These findings lay a solid foundation for further study of roles of HM448398 using knock-in and RNA interference methods during liver regeneration.

Key words: Homologue of Serpina3n; Prokaryotic expression; Liver regeneration; Electronic clone; Expression change

\section{INTRODUCTION}

The liver is a unique organ in that it has the capability of regenerating (Yokoyama et al., 2007). Liver regeneration (LR) is a process during which the liver recovers its mass and functions after damage owing to various causes such as partial hepatectomy ( $\mathrm{PH}$ ) (Higgins and Anderson, 1931; Kountouras et al., 2001). LR involves a sequence of well-orchestrated changes in physiological activities, such as cell activation, hepatocyte proliferation, redifferentiation, and rebuilding of tissue structure (Guo et al., 2006; Michalopoulos, 2010). The study of LR is focused on using subtractive hybridization and complementary DNA (cDNA) microarray for the screening and cloning of LR-related genes and the clarification of their roles in modulating LR. Suppression subtractive hybridization ( $\mathrm{SSH}$ ), the best known subtraction method, can effectively separate and identify low-abundance differentially expressed genes (Liu et al., 2001). To identify additional specific LR-related genes and elucidate the mechanisms of LR, Xu et al. $(2001,2005)$ established the short interval successive partial hepatectomy (SISPH) model, constructed a 4-8 h forward subtractive cDNA library of 0-4-8-12 h, and screened several differentially expressed sequence tags (ESTs). Of these ESTs, Ab1355 (GenBank accession No. CD052215) was found to be dramatically upregulated during LR, and Nucleotide Basic Local Alignment Search Tool (BLASTN) searching indicated that it had no significant homology with known rat cDNAs, suggesting that it may represent a new gene. In this study, we predicted the open reading frame (ORF) sequence using an in silico cloning method, amplified the ORF, constructed it into pGEX-2TK vector to induce the expression of recombinant protein, purified the recombinant protein, prepared a polyclonal antibody, and analyzed its expression profile during LR. The results lay a foundation for further elucidation of its role in LR.

\section{MATERIAL AND METHODS}

\section{Rat models of PH and SISPH}

Adult healthy male Sprague-Dawley rats weighing $210 \pm 20 \mathrm{~g}$ were supplied by the Experimental Animal Center of Henan Normal University and housed in a controlled-temperature room $\left(22^{\circ} \pm 1^{\circ} \mathrm{C}\right)$ with a 12:12-h light:dark cycle (light period from 6:00 am to 18:00 $\mathrm{pm})$. The rats were fed standard rodent chow and allowed free access to distilled water. According to a method described by Xu et al. (2001), the lobus external sinister, lobus centralis sinister, lobus centralis, and lobus dexter were removed one by one at 4 time points of 0,4 , 
8 , and $12 \mathrm{~h}$, a procedure called $0-4-8-12 \mathrm{~h}$ SISPH. A total of 40 male rats were taken and randomly divided into 10 groups with 4 rats in each: 9 PH groups and 1 normal control group. $\mathrm{PH}$ groups were subjected to $2 / 3 \mathrm{PH}$ as described by Higgins and Anderson (1931). Four rats were anesthetized and killed at $0,2,6,12,24,30,36,72,120$, and $168 \mathrm{~h}$ after PH. Four sections of approximately $5 \times 5 \times 2-3 \mathrm{~mm}$ were cut from the middle of the right lobe of the liver and fixed in $10 \%$ neutral-buffered formalin for immunochemical analysis. The remaining liver tissues were collected in RNase-free tubes and stored at $-80^{\circ} \mathrm{C}$ until RNA extraction. All handling procedures were conducted in compliance with current animal protection laws in China.

\section{In silico cloning of the full-length ORF}

The EST sequence (CD052215) was subjected to BLASTN searching against the rat genome (http://www.ncbi.nlm.nih.gov/genome/guide/rat/index.html) and was mapped to rat chromosome $6 \mathrm{q} 32$ via electronic polymerase chain reaction (PCR). Two clone sequences (GenBank accession Nos. NW_047762.2 and NW_001084846.1) on chromosome 6q32 were obtained that showed high sequence homology with the above EST. We predicted the exons and introns in the homologous region of NW_047762.2 and NW_001084846.1 using the GENESCAN (http://genes.mit.edu/GENSCAN.html) and BLAST 2 sequencing programs and then assembled the predicted exons into the coding sequence. The ORF was of $2097 \mathrm{bp}$ composed of 9 exons, which encoded a protein of 698 amino acids.

\section{Isolation of total RNA and cloning of the full-length ORF}

To obtain the full-length ORF, we designed a pair of gene-specific primers with Primer Express 2.0 according to the messenger RNA (mRNA) sequences of the predicted ORF sequence (forward primer: 5'-ATGGCCTTCATTGCAGCTTTG-3'; reverse primer: 5'-TCAATGCCTGGGGAGAAGCG-3'). Total RNA of the regenerating rat liver $8 \mathrm{~h}$ after PH was extracted with the Trizol reagent (Invitrogen, Carlsbad, CA, USA) according to manufacturer instructions and purified following the RNeasy Mini protocol (Qiagen, USA). The concentration and purity of total RNA were determined using a bio-photometer (Eppendorf, Germany) and 1.2\% agarose electrophoresis (180 V, $0.5 \mathrm{~h}$ ) (Arkin et al., 1998). Total RNA $(2 \mu \mathrm{g})$ was reverse-transcribed using random primers and a Reverse Transcription Kit (Promega, USA). A first-strand cDNA sample was subjected to PCR amplification, and the PCR cycling conditions were modified to $95^{\circ} \mathrm{C}$ for 5 min followed by 30 cycles for $30 \mathrm{~s}$ at $95^{\circ} \mathrm{C}, 30 \mathrm{~s}$ at $60^{\circ} \mathrm{C}$, and $2 \mathrm{~min}$ at $72^{\circ} \mathrm{C}$. The PCR products were separated with electrophoresis on $1.5 \%$ agarose gels and visualized through scanning with an ultraviolet imaging system (Gucun, Shanghai, China). To assess the specificity of the amplification, we had the obtained band subcloned into pGEM-T vector and sequenced (Shanghai Generay Biotech, Shanghai, China).

\section{Construction of the prokaryotic expression vector of the full-length ORF}

The ORF sequence was amplified again with the specific primers and Bam HI or EcoRI enzyme sites (forward primer: 5'-ATATGGATCCATGGCCTTCATTGC-3'; reverse primer: 5'-TGTGAATTCTCAATGCCTGGGGAG-3'). The obtained band of PCR products 
was inserted into pGEX-2TK vector using BamHI and EcoRI and then transfected into BL21. The positive clones were assayed through PCR analysis with the expression-specific primers and the common primers for pGEX-2TK and then sequenced by Shanghai Generay Biotech.

\section{Bacterial expression and purification of the recombinant Serpina3n homologous protein}

One positively identified clone containing the recombinant plasmids (pGEX-2TKglutathione-S-transferase [GST]-ORF) was cultivated with shaking in $10 \mathrm{~mL}$ Luria-Bertani broth (containing $50 \mu \mathrm{g} / \mathrm{mL}$ ampicillin) at $37^{\circ} \mathrm{C}$ overnight. Then, $1 \mathrm{~mL}$ bacteria strain BL21 was removed and cultivated in $100 \mathrm{~mL}$ Luria-Bertani broth (containing ampicillin as described above) with shaking until the optical density reached 0.6-0.8. Protein expression was induced with isopropyl-D-thiogalactopyranoside (IPTG; Sigma, USA), and parameters such as IPTG concentration, inducible time, and temperature were optimized (Liu et al., 2005). After induction with IPTG at the appropriate temperature, the bacteria were pelleted with centrifugation at $4000 \mathrm{~g}$ for $20 \mathrm{~min}$ at $4^{\circ} \mathrm{C}$. The pellets were then suspended in phosphate-buffered saline at $4^{\circ} \mathrm{C}$ and further subjected to sonication on ice with a W-375 sonication ultrasonic processor (Heat Systems-Ultrasonics Inc., NY, USA) until the lysate solution became clear. The soluble supernatant was collected through centrifugation, whereas insoluble sediment was resuspended with $5 \mathrm{~mol} / \mathrm{mL}$ urea. Finally, they were further subjected to sodium dodecyl sulfate-polyacrylamide gel electrophoresis (SDS-PAGE) to verify the expression of recombinant proteins.

To purify the recombinant protein, we added $2 \mathrm{~mL}$ of a $50 \%$ slurry of GlutathioneSepharose 4B (Amersham Pharmacia Biotech, Peapack, NJ, USA) to $100 \mathrm{~mL}$ lysate supernatant, incubated the mixture for $30 \mathrm{~min}$ at room temperature, and centrifuged it at $500 \mathrm{~g}$ for $5 \mathrm{~min}$. The sediment was washed 3 times with a 10 -fold volume of phosphate-buffered saline at $4{ }^{\circ} \mathrm{C}$, resuspended in a 1-fold volume of elution solution, and incubated with shaking for 10 $\mathrm{min}$ at room temperature. After centrifugation at $500 \mathrm{~g}$ for $5 \mathrm{~min}$, the supernatant of the elution solution was pooled into a new Eppendorf tube. Elution was repeated twice, and SDS-PAGE was carried out to analyze the supernatant from each elution. The protein concentration was determined using the Bradford method (Ma and Zhao, 2007). We used the purified recombinant Serpina3n protein as an antigen to immunize rabbits for the production of polyclonal antibodies and prepared an antibody with high specificity and sensitivity as described by Li et al. (2010).

\section{Immunochemical analysis}

Small cubes approximately $5 \times 5 \times 2-3 \mathrm{~mm}$ from the right lobe of the liver were fixed with $10 \%$ neutral-buffered formalin for $24 \mathrm{~h}$ and washed with tap water for $24 \mathrm{~h}$. They were then routinely dehydrated with a graded series of ethanol, cleared in xylene, embedded in paraffin, and sectioned at $5-\mu \mathrm{m}$ thicknesses. The slices were stained with hematoxylin for 3 min, immersed in ammonia water, $\mathrm{pH} 8.0$, for $30 \mathrm{~s}$, and counterstained with $0.5 \%$ eosin for 5 min. Finally, they were dehydrated using gradient ethanol, cleared in xylene, and sealed with a neutral gum. Histopathologic examinations of the sections were conducted by a pathologist and then peer reviewed. The sections were incubated separately with a 1:200 dilution $(\mathrm{v} / \mathrm{v})$ of albumin and catalytic glucose-6-phosphatase antibodies overnight at $4{ }^{\circ} \mathrm{C}$ and then with a 1:5000 (v/v) diluted biotin-labeled secondary antibody at $37^{\circ} \mathrm{C}$ for $60 \mathrm{~min}$. The system was hybridized with streptavidin-biotin complex at $37^{\circ} \mathrm{C}$ for $30 \mathrm{~min}$. All steps were performed at 
room temperature. The paraffinized sections of liver tissue at the corresponding time points were prepared for hematoxylin and eosin staining and immunohistochemical analysis. The results were observed and analyzed under an optical microscope (Horn et al., 2008).

\section{Fluorescent quantitative reverse transcription (RT)-PCR}

To verify the expression change of the Serpina3n homologue at the mRNA level during LR, fluorescent quantitative RT-PCR was performed. Primer sequences were designed using Primer Express 2.0 according to the mRNA sequences of the Serpina3n homolog and the $\beta$-actin gene (internal control) and synthesized by Shanghai Generay Biotech. The primers used were as follows: forward primer for the target gene: 5'-GAGACAGGCACAGAAGCAGC-3'; reverse primer for the target gene: 5'-AGCGTCAACTCTTGGGAAGT-3'; forward primer for the $\beta$-actin gene internal control: 5'-ACATCCGTAAAGACCTCTATGCCAACA-3'; reverse primer for the $\beta$-actin gene: 5'-GTGCTAGGAGCCAGGGCAGTAATCT-3'. The total RNA of the regenerating rat livers at 10 time points after $\mathrm{PH}$ was extracted and purified as described above. Before RT, contaminating genomic DNA was removed using DNaseI (Promega). Total RNA $(2 \mu \mathrm{g})$ was reverse-transcribed using random primers and a Reverse Transcription Kit (Promega). First-strand cDNA samples were subjected to quantitative PCR amplification using SYBR ${ }^{\circledR}$ Green I on the Rotor-Gene 3000A (Corbett Robotics, Brisbane, Australia). The PCR cycling conditions for each gene were modified to $95^{\circ} \mathrm{C}$ for 2 min followed by 40 cycles for $15 \mathrm{~s}$ at $95^{\circ} \mathrm{C}, 15 \mathrm{~s}$ at $60^{\circ} \mathrm{C}$, and $30 \mathrm{~s}$ at $72^{\circ} \mathrm{C}$. Every sample was analyzed in triplicate. Standard curves were generated from 5 repeated 10-fold serial dilutions of cDNA, and the copy numbers of the target genes in every milliliter of the sample were calculated according to their standard curves (Wang and $\mathrm{Xu}, 2010 \mathrm{~b}$ ).

\section{RESULTS}

\section{Cloning and sequence analysis of the full-length ORF}

The EST of Ab1355 (GenBank accession No. CD052215) was screened from the 4-8 $\mathrm{h}$ forward subtractive cDNA library of 0-4-8-12 h. Therefore, the regenerating rat liver at $8 \mathrm{~h}$ of 0-4-8-12 h SISPH was obtained as the object of this study. As displayed in Figure 1A, the ratio of the amounts of $28 \mathrm{~S}$ and $18 \mathrm{~S}$ ribosomal RNA was 2:1, and the total RNA was regarded as a qualified sample for the following RT and cDNA amplication. Using the specific primers designed for the predicted ORF, we amplified one bright fragment from $8 \mathrm{~h}$ regenerating liver tissue, and its molecular weight was greater than $1000 \mathrm{bp}$ (Figure 1B). After insertion into pGEM-T vector and sequencing, the product was found to be $1293 \mathrm{bp}$ long and was deposited in GenBank (accession No. HM448398). Using DNAman to compare the sequences of HM448398 and the predicted ORF, we found that the real product was 5 exons shorter than predicted (Figure 2), suggesting that the 2 ORFs may be different transcripts of one gene. The results showed that the in silico method of cloning was not fully reliable, although it provided one method for predicting the full-length of one cDNA. HM448398 had high sequence homology with rat Serpina3n, with $92.69 \%$ sequence identity with rat Serpina3n in the BLASTN search, suggesting that it may be a homolog of Serpina3n. Thus, we considered the full-length ORF a Serpina3n homologous gene in our study. 
A

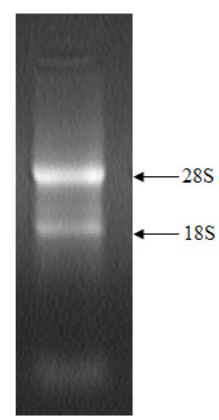

B

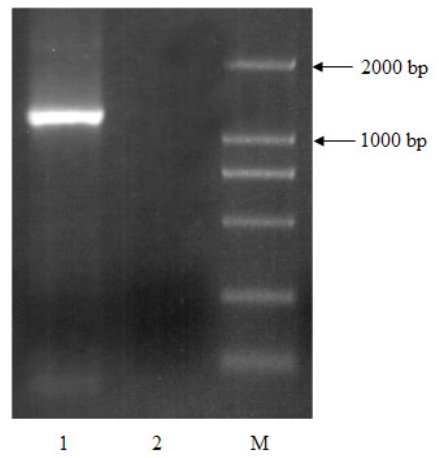

Figure 1. Amplification of full-length open reading frame (ORF) by RT-PCR. A. Denaturing agarose gel electrophoresis of total RNA from an 8-h regenerating liver in 0-4-8-12 h SISPH. B. Detection of amplification product. Lane 1 = Full-length ORF; lane 2 = negative control; lane $M=$ DNA marker.

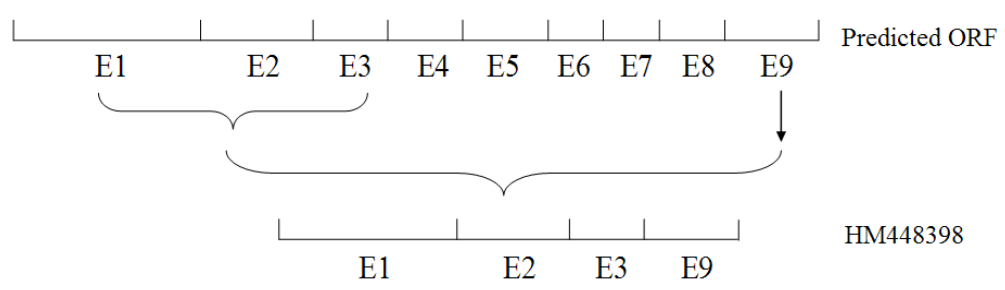

Figure 2. Alignment map of the predicted open reading frame (ORF) and HM448398. E represents expressed open reading framework.

\section{Identification of the recombinant prokaryotic expression vector}

PCR analysis was applied to assay 2 randomly selected clones, and the results showed that the full-length of the ORF was contained in the pGEX-2TK vector (Figure 3). Sequencing results indicated that the ORF was the same as the sequence when it was constructed into a pGEM-T vector, implying that the recombinant vector pGEX-2TK-HM448398 was successfully constructed.

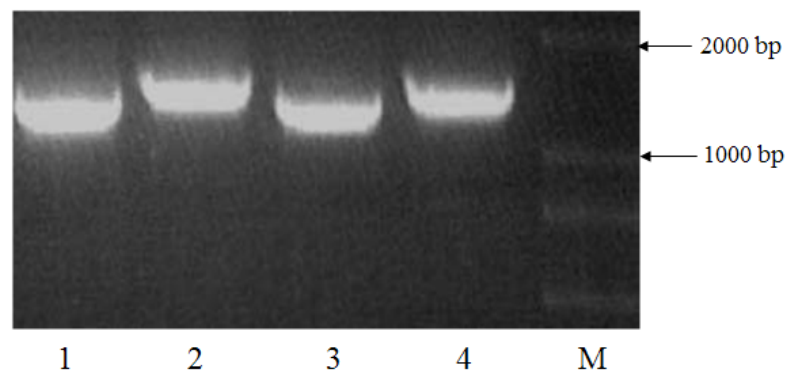

Figure 3. PCR detection results of recombinant prokaryotic expression plasmids. Lanes 1 and $3=$ Amplification result of clones 1 and 2 used in specific primers; lanes 2 and $4=$ amplification result of clones 1 and 2 used in universal primers; lane $M=$ DNA marker. 


\section{Induced expression and purification of the GST-fused Serpina3n homologous protein}

After optimizing parameters such as IPTG concentration, inducible time, and temperature, we found that the GST-fused protein was expressed effectively when the bacterium strain BL21 was induced overnight with $50 \mu \mathrm{M}$ IPTG at $20^{\circ} \mathrm{C}$ and subjected to $120 \mathrm{rpm}$ for 8-9 h after cultivation with shaking until the optical density reached 0.6-0.8. SDS-PAGE analysis demonstrated that a large amount of protein with an approximate molecular weight of $71 \mathrm{kDa}$ appeared in the supernatant, whereas less protein was present in the sediment. This result implied that the GST-fused protein was expressed mainly as soluble protein when induced at optimal conditions (Figure 4A). Afterward, BL21 was cultivated and induced with IPTG on a large scale. The lysate supernatant was purified with Glutathione-Sepharose 4B, and SDS-PAGE analysis showed that the GST-fused protein was effectively purified in a high quantity (Figure 4B). The concentration of the purified GST-fused protein was assayed as 1.4 $\mu \mathrm{g} / \mu \mathrm{L}$ in this study.

A

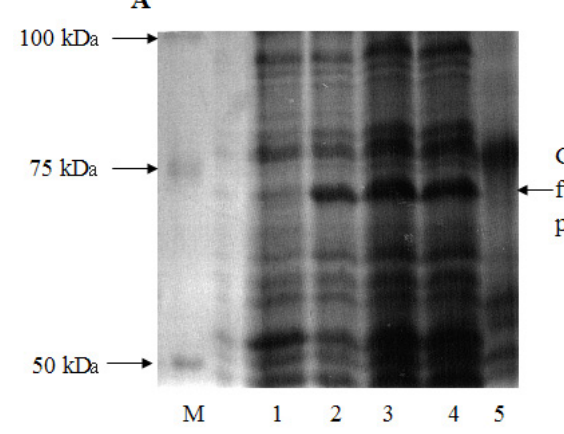

B

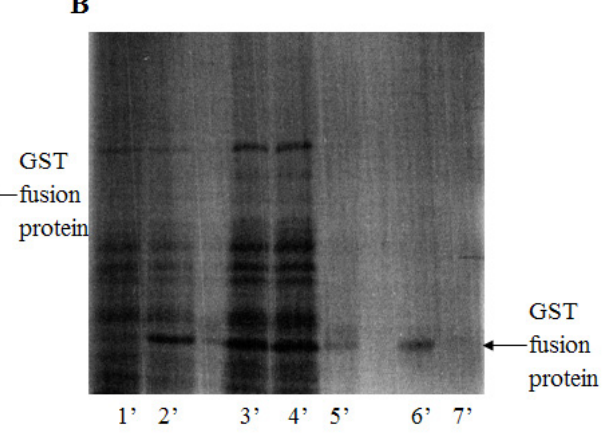

Figure 4. SDS-PAGE analysis of the glutatione-S-transferase (GST)-fused Serpina3n homologous protein expressed in BL21. A. Lane $M=$ DNA marker; lane $1=$ control whole bacteria; lane 2 = induced whole bacteria; lane 3 = induced whole bacteria after ultrasonication; lane $4=$ induced supernatant after ultrasonication; lane $5=$ induced pellets after ultrasonication. B. Lane 1' = control whole bacteria; lane 2' = induced whole bacteria; lane 3' = induced whole bacteria after ultrasonication; lane 4' = induced supernatant after ultrasonication; lane 5' = induced pellets after ultrasonication; lanes 6' and 7' = elution products for the first and second time.

\section{Expression change of the Serpina3n homologous protein during LR}

After the polyclonal antibody was primarily purified, we used immunohistochemistry to analyze the expression changes of the rat Serpina3n homolog at 10 recovery time points after PH. We demonstrated that the increased expression of the Serpina3n homolog became evident at 6-24 h, with the first expression peak at $12 \mathrm{~h}$. After 30-36 h, expression reached the lowest point. Afterwards, expression was again strikingly enhanced at $72 \mathrm{~h}$ and then decreased until $168 \mathrm{~h}$. Moreover, the stain-positive granules were mainly located in the nucleus, suggesting that the protein of the Serpina3n homolog is a nucleoprotein (Figure 5).

\section{Expression trend of the Serpina3n homologous gene during LR}

Fluorescent quantitative RT-PCR was applied to assay the expression changes of the 
Serpina3n homologous gene during LR, and the results showed that the mRNA level exhibited rapid increase at $2 \mathrm{~h}$ and first peaked $6 \mathrm{~h}$ after PH. Its mRNA level slowly decreased and reached its lowest point at 30-36 h, then again increased and peaked at $72 \mathrm{~h}$ followed by slow downregulation until the end of the observation (168 h; Figure 6).
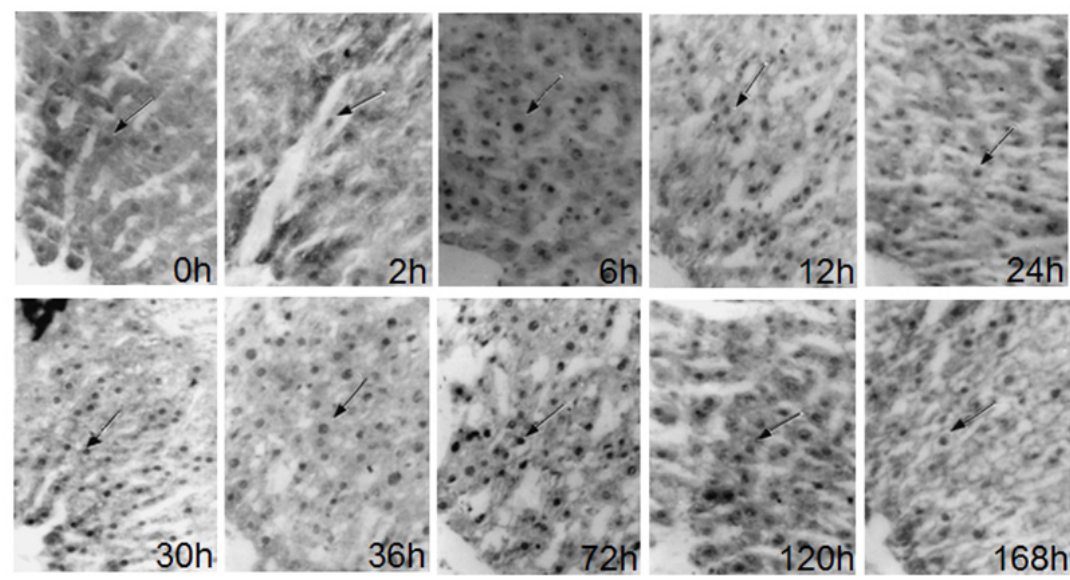

Figure 5. Expression change of the Serpina3n homologous protein at different recovery time points after partial hepatectomy detected by immunohistochemistry (200X).

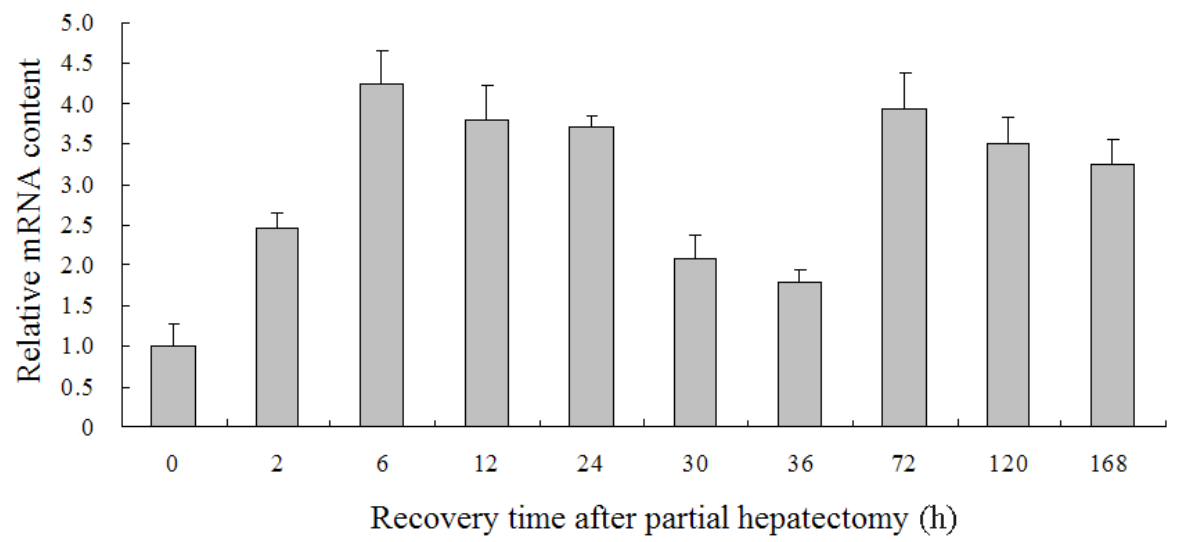

Figure 6. mRNA expression level of the Serpina3n homologous gene at different recovery time points after partial hepatectomy detected by quantitative RT-PCR.

\section{DISCUSSION}

The liver is one of the vital organs of the human body, and its powerful regenerative capacity has become a hot topic for scientific study. Generally, the mechanism of LR is studied using regenerating liver tissues after $2 / 3$ hepatectomy. The LR process is divided into 2 ways: according to cellular physiological and biochemical activities into a priming phase $(0-6 \mathrm{~h}$ after $\mathrm{PH})$, a progressive phase (6-72 h after $\mathrm{PH})$, and a terminal phase (72-168 h after $\mathrm{PH})$ (Zheng 
et al., 2009) and according to time course into a forepart (0.5-4 h after $\mathrm{PH})$, prophase (6-12 $\mathrm{h}$ after $\mathrm{PH})$, metaphase (12-72 $\mathrm{h}$ after $\mathrm{PH}$ ), and anaphase (72-168 $\mathrm{h}$ after $\mathrm{PH})$. LR involves various physiological and biochemical activities such as cell activation, de-differentiation, proliferation and its regulation, re-differentiation, and rebuilding of structure and function.

To seek novel differential display genes responsible for LR, we used SSH to obtain a bulk of upregulated and downregulated ESTs in regenerating rat liver. Our previous study had used 4- and 8-h regenerated livers as experimental materials from which to extract mRNA and reverse transcribe it to cDNA and then apply SSH to construct a forward-subtracted cDNA library, which included some upregulated ESTs expressed in 0-4-8-12 h SISPH (Xu et al., 2005). Of these ESTs, Ab1355 (GenBank accession No. CD052215) was dramatically upregulated during LR, and BLASTN searching indicated that it had no significant homology with known rat cDNAs, suggesting that it might represent a new gene. In this study, we predicted the ORF sequence corresponding to CD052215 using an in silico cloning method and designed a pair of specific primers to clone the full-length ORF. A full-length ORF was cloned from an 8-h regenerating rat liver and deposited in GenBank (accession No. HM448398). Through sequence analysis of HM448398 and the predicted ORF, we found that the 2 ORFs might be different transcripts of a gene. The sequence of HM448398 was highly similar to that of rat Serpina3n, suggesting that it may be a homolog of Serpina3n. Although the predicted result of in silico cloning was, to some extent, different from the actual product, in silico cloning was an efficient method for obtaining the full-length sequence of a gene.

The expression vector for HM448398 was constructed by ligating the amplified fragment to the expression vector pGEX-2TK, which was used to transform Escherichia coli strain BL21. After induction with IPTG, BL21 expressed the corresponding protein with high quality and purity. The pGEX-2TK vector is a highly efficient prokaryotic expression vector with an IPTG-induced promoter, and the expressed target protein has a characteristic GST sequence at the N-terminal that can effectively help purifying the target protein using affinity chromatography. In this study, the predicted molecular weight for the Serpina3n homologous protein was $45 \mathrm{kDa}$, and the molecular weight of the GST-fused protein was expected to be $71 \mathrm{kDa}$ after adding the molecular weight of GST ( $26 \mathrm{kDa}$ into that of Serpina3n homolog). These estimates were consistent with the results of SDS-PAGE. Moreover, we needed to compare the conditions and select the one that induced high-quality protein because the optimal conditions are usually unique (Liu et al., 2005). Owing to the difficulties of purifying expressed proteins from inclusion bodies, we made great efforts to select the optimal induction concentration, time, and temperature. A high-quality soluble GST-fused target protein was ultimately obtained.

HM448398 is highly homologous to rat Serpina3n in sequence, suggesting that it may be a member of the serpin (serine protease inhibitor) superfamily. Immunohistochemistry and real-time RT-PCR were applied to analyze the expression changes of the Serpina3n homologue, and we found that mRNA and protein levels of the homologue were significantly changed with similar expression trends during LR. Furthermore, the upregulation of the Serpina3n homologous protein lagged slightly behind that of its mRNA, implying that the increased mRNA level of the Serpina3n homologue could partly account for the enhanced expression of the Serpina3n homologous protein. Certain serine protease inhibitory factors are upregulated at the priming stage of LR (Deng et al., 2009) and may participate in antagonizing inflammatory or acute phase reactions after $\mathrm{PH}$, thus preventing further damage to the 
remnant liver (Marchbank et al., 2009; Wang and Xu, 2010a), which could represent a type of endogenous cell protection mechanism. In addition, some studies have demonstrated that serine protease inhibitory factors are involved in the regulation of serine protease activation after nerve injury or the regulation of hepatocyte growth factor after liver damage and then play a vital role in acute phase reactions after hepatectomy and balancing the LR process (Gesase and Kiyama, 2007; Jin, 2007). This study showed that the protein and mRNA of the Serpina3n homologue were expressed significantly in the priming and termination phases of LR. Therefore, we speculate that the Serpina3n homologue may have a regulatory role in acute phase reactions and LR processes.

In summary, a rat Serpina3n homologous gene was successfully cloned, inserted into a prokaryotic expression vector, and induced to express a high-quality protein by an inducer at optimal conditions. After purification of the GST-fused protein and preparation of polyclonal antibody to the rat Serpina3n homolog, the expression dynamics and pattern of its protein and mRNA during LR were further analyzed. The results of this study lay a solid foundation for further study of the roles of this gene in LR using knock-in and RNA interference methods.

\section{ACKNOWLEDGMENTS}

Research supported by the Henan Scientific and Technological Research Projects (\#082300450550, \#112300410275, \#112102310652, and \#122300410339), the Natural Science Project of the Education Department of Henan Province (\#2010A350002) and the Doctoral Scientific Research Start-up Foundation of Henan Normal University (\#11128).

\section{REFERENCES}

Arkin A, Ross J and McAdams HH (1998). Stochastic kinetic analysis of developmental pathway bifurcation in phage lambda-infected Escherichia coli cells. Genetics 149: 1633-1648.

Deng X, Li W, Chen N, Sun Y, et al. (2009). Exploring the priming mechanism of liver regeneration: proteins and protein complexes. Proteomics 9: 2202-2216.

Gesase AP and Kiyama H (2007). Peripheral nerve injury induced expression of mRNA for serine protease inhibitor 3 in the rat facial and hypoglossal nuclei but not in the spinal cord. Ital. J. Anat. Embryol. 112: 157-168.

Guo F, Nian H, Zhang H, Huang L, et al. (2006). Proteomic analysis of the transition from quiescent to proliferating stages in rat liver hepatectomy model. Proteomics 6: 3075-3086.

Higgins GM and Anderson RM (1931). Experimental pathology of the liver: restoration of the liver of the white rat following partial surgical removal. Arch. Pathol. 12: 186-202.

Horn TL, Harder JB, Johnson WD, Curry PT, et al. (2008). Integration of in vivo and in vitro approaches to characterize the toxicity of Antalarmin, a corticotropin-releasing hormone receptor antagonist. Toxicology 248: 8-17.

Jin ZZ (2007). The research progress on the relationship between hepatocyte growth factor and liver injury. J. Med. Sci. Yanbian Univ. 30: 307-309.

Kountouras J, Boura P and Lygidakis NJ (2001). Liver regeneration after hepatectomy. Hepatogastroenterology 48: 556-562.

Li H, Zhang W, Sheng M, Li W, et al. (2010). Cloning, expression, purification of spinach carboxyl-terminal processing protease of D1 protein with hydrolysis activity and preparation of polyclonal antibody. Sheng Wu Gong Cheng Xue Bao 26: 495-502.

Liu ZW, Zhao MJ and Li ZP (2001). Identification of up-regulated genes in rat regenerating liver tissue by suppression subtractive hybridization. Sheng Wu Hua Xue Yu Sheng Wu Wu Li Xue Bao 33: 191-197.

Liu ZY, Zhang FC, Wang Y and Lv GD (2005). Expression of the antifreeze protein gene of a pyrochroid beetle Dendroides canadensis in prokaryote and detection of the protein biological activity. Acta Entomol. Sin. 48: 179-183.

Ma H and Zhao XY (2007). Construction of pGEX-2TK-Hsbp1 prokaryotic expression vector and expression of HSBP1. J. Zhengzhou Coll. Anim. Husb. Eng. 27: 4-6.

Marchbank T, Weaver G, Nilsen-Hamilton M and Playford RJ (2009). Pancreatic secretory trypsin inhibitor is a major 
motogenic and protective factor in human breast milk. Am. J. Physiol. Gastrointest. Liver Physiol. 296: G697-G703. Michalopoulos GK (2010). Liver regeneration after partial hepatectomy: critical analysis of mechanistic dilemmas. Am. J. Pathol. 176: 2-13.

Wang GP and Xu CS (2010a). Pancreatic secretory trypsin inhibitor: More than a trypsin inhibitor. World J. Gastrointest. Pathophysiol. 1: 85-90.

Wang GP and Xu CS (2010b). Reference gene selection for real-time RT-PCR in eight kinds of rat regenerating hepatic cells. Mol. Biotechnol. 46: 49-57.

$\mathrm{Xu}$ CS, Li YH, Duan RF and Lu AL (2001). Effects of the short interval successive partial hepatectomy on rat survival and liver tissue structure. Dong Wu Xue Bao 47: 659-665.

Xu CS, Yuan JY, Li WQ, Han HP, et al. (2005). Identification of expressed genes in regenerating rat liver in 0-4-8-12 h short interval successive partial hepatectomy. World J. Gastroenterol. 11: 2296-2305.

Yokoyama Y, Nagino M and Nimura Y (2007). Mechanisms of hepatic regeneration following portal vein embolization and partial hepatectomy: a review. World J. Surg. 31: 367-374.

Zheng ZY, Weng SY and Yu Y (2009). Signal molecule-mediated hepatic cell communication during liver regeneration. World J. Gastroenterol. 15: 5776-5783. 